\title{
Accuracy of EUS-FNA in Solid Pancreatic Lesions: Sometimes Size Does Matter
}

\author{
Tilak Shah ${ }^{1,2} \cdot$ Alvin M. Zfass ${ }^{1,2}$ \\ Published online: 6 February 2019 \\ (c) This is a U.S. Government work and not under copyright protection in the US; foreign copyright protection may apply 2019
}

Exocrine pancreatic cancer carries an extremely poor prognosis; although surgical resection is the sole potentially curative option, only $15-20 \%$ of patients are candidates for pancreatectomy [1]. Even among patients who undergo surgery, median survival is only 10-20 months [1]. Yet, in the limited subgroup of patients with tumors $<1 \mathrm{~cm}$ and without any malignant adenopathy, 5-year survival after surgery can be as high as $50 \%$ [2].

Mortality from pancreatic surgery has declined substantially over the last several decades, although morbidity remains high at $40-50 \%$, even in high-volume centers [3]. Complications such as delayed gastric emptying (17\% of patients), bile leak (1-2\%), and pancreatic fistula (5-10\%) can significantly impact quality of life [3]. Besides exocrine pancreatic adenocarcinoma, the differential diagnosis in patients with solid pancreatic lesions (SPLs) includes other neoplasms such as pancreatic neuroendocrine tumor [PNET], metastasis, and lymphoma, as well as benign conditions such as chronic pancreatitis and perivascular epithelioid cell tumors. Pancreatic surgery does not typically provide any survival or quality-of-life benefit for PNETs $<1 \mathrm{~cm}$, pancreatic metastases, pancreatic lymphoma, or minimally symptomatic benign conditions. Thus, while pancreatic surgery offers the only hope for survival for a small exocrine cancer, unnecessary surgery can cause considerable harm, elevating the importance of accurate tumor diagnosis.

Investigators have tested a plethora of biomarkers for the early detection of pancreatic adenocarcinoma such as circulating tumor cells, autoantibodies, epigenetic markers, and miRNA. To date, none have demonstrated sufficient

Tilak Shah

Tilak.Shah@va.gov

1 Division of Gastroenterology, Hunter Holmes McGuire Veterans Affairs Medical Center, 111 N, 1201 Broad Rock Blvd, Richmond, VA 23224, USA

2 Division of Gastroenterology, Virginia Commonwealth University Health System, Richmond, VA, USA sensitivity and specificity to justify incorporation into routine clinical practice. Diagnosis depends primarily on imaging and tissue diagnosis, specifically on cytologic specimens obtained during endoscopic ultrasound (EUS)guided fine needle aspiration (FNA). In this issue of Digestive Diseases and Sciences, Sugiura et al. [4] retrospectively assessed the accuracy of EUS-FNA (up to eight passes) in 761 patients with 788 SPLs. The pancreatic mass was $<1 \mathrm{~cm}$ in 36 lesions and 1-2 cm in 223 lesions. Patients with cystic lesions were excluded, as were procedures performed using core needles. Rapid on-site evaluation (ROSE), that can provide immediate feedback regarding the adequacy of FNA-acquired samples, was performed, but cell block preparation (see below) was not available. Sensitivity of EUSFNA was $<90 \%$ in lesions $<1 \mathrm{~cm}, 95 \%$ for lesions $1-2 \mathrm{~cm}$, and $>95 \%$ for lesions $>2 \mathrm{~cm}$. Specificity of EUS-FNA was $100 \%$ irrespective of mass size. False-negative EUS-FNA was documented in 3/36 lesions (two neuroendocrine tumors and one metastatic renal cancer). The final diagnosis was pancreatic adenocarcinoma in only $5 / 36$ lesions $<1 \mathrm{~cm}$, all of which were correctly identified with EUS-FNA. Using multivariate analysis, pancreatic mass size was the only factor affecting the accuracy of EUS-FNA.

With regard to overall accuracy, these results are consistent with those of $>40$ previously published studies that evaluated the accuracy of EUS-FNA for SPLs [5]. Pooled analyses report a high specificity ( $>95 \%$ ), but lower sensitivity $(<90 \%)$ [5]. Similar to the current study, other retrospective studies have also documented lower sensitivity of EUS-FNA in lesions $<1 \mathrm{~cm}$. For instance, Haba et al. [6] reported a sensitivity of $73 \%$ if the lesion was $<1 \mathrm{~cm}$ $(n=40)$, but $94 \%$ if the lesion was $>2 \mathrm{~cm}$. In a retrospective study that assessed EUS-FNA accuracy in 583 SPLs, Siddiqui et al. [7] found EUS-FNA sensitivity was a dismal $47 \%$ for SPLs $<1 \mathrm{~cm}$.

Has the limit been reached when it comes to EUS accuracy for SPLs? EUS accuracy relies on several factors, including tissue acquisition techniques, tissue acquisition 
tools, tissue preparation techniques, and cytopathologic interpretation. The literature reports several tissue acquisition techniques (e.g., fanning, slow capillary pull through, wet suction, and the "knock door" technique), with conflicting results for improvements in accuracy [8]. "Core" needles with differing designs (e.g., reverse bevel, fork tip and Franseen geometry) are now commercially available. Since data regarding their accuracy continue to accumulate, a consensus will build regarding the relative merits of these needle designs with regard to the diagnostic yield for small SPLs. Some investigators have reported improved accuracy if the acquired tissue is processed into a cell block, where cellular material obtained during FNA is centrifuged into a concentrated cell pellet that is processed through routine histologic preparation. The theoretical advantage is that the pathologist can cut the cell block into sections for microscopic examinations and ancillary testing. Unfortunately, prospective randomized trials comparing cell block to ROSE acquired for SPLs are lacking. Even when the specimen is adequate, cytopathologic interpretation is often challenging - pathology reports of "atypical" cells are the bane of the endosonographer's existence. There are multiple publications addressing the use of artificial intelligence using neural networks for cervical cytopathologic interpretation [9]. The development of such algorithms for SPLs could potentially reduce subjectivity and bias in cytopathology interpretation.

In summary, the current study corroborates data from existing publications that EUS-FNA is highly specific and is reasonably sensitive in small SPLs. Given the high stakes, "reasonably sensitive" is often not good enough. Perhaps advances related to some (or all) of these factors can "move the needle" toward increasing the sensitivity of EUS-FNA in small SPLs.

Author's contribution Dr. Shah wrote the article, and Dr. Zfass edited the article.

Funding Dr. Shah receives funding from the American Society for Gastrointestinal Endoscopy (ASGE). The content is solely the responsibility of the author and does not necessarily represent the official views of the ASGE.

\section{Compliance with ethical standards}

Conflicts of interest No author has relevant conflicts of interest.

Disclosures Shah is the guarantor of this article.

\section{References}

1. Clancy TE. Surgery for pancreatic cancer. Hematol Oncol Clin North Am. 2015;29:701-716.

2. Benassai G, Mastrorilli M, Quarto G, et al. Survival after pancreaticoduodenectomy for ductal adenocarcinoma of the head of the pancreas. Chir Ital. 2000;52:263-270.

3. Pecorelli N, Nobile S, Partelli S, et al. Enhanced recovery pathways in pancreatic surgery: state of the art. World J Gastroenterol. 2016;22:6456-6468.

4. Suguira R, Kuwatani M, Koji H. Effect of pancreatic mass size on clinical outcomes of endoscopic ultrasound-guided fine-needle aspiration. Dig Dis Sci. (Epub ahead of print). https://doi. org/10.1007/s10620-018-5435-3.

5. Puli SR, Bechtold ML, Buxbaum JL, Eloubedi MA. How good is endoscopic ultrasound-guided fine-needle aspiration in diagnosing the correct etiology for a solid pancreatic mass? A meta-analysis and systematic review. Pancreas. 2013;42:20-26.

6. Haba S, Yamao K, Bhatia V, et al. Diagnostic ability and factors affecting accuracy of endoscopic ultrasound-guided fine needle aspiration for pancreatic solid lesions: Japanese large single center experience. J Gastroenterol. 2013;48:973-981.

7. Siddiqui AA, Brown LJ, Hong SK, et al. Relationship of pancreatic mass size and diagnostic yield of endoscopic ultrasoundguided fine needle aspiration. Dig Dis Sci. 2011;56:3370-3375.

8. Yamabe A, Irisawa A, Bhutani MS, et al. Efforts to improve the diagnostic accuracy of endoscopic ultrasound-guided fineneedle aspiration for pancreatic tumors. Endosc Ultrasound. 2016;5:225-232.

9. Pouliakis A, Karakitsou E, Margari N, et al. Artificial neural networks as decision support tools in cytopathology: past, present, and future. Biomed Eng Comput Biol. 2016;7:1-18. 